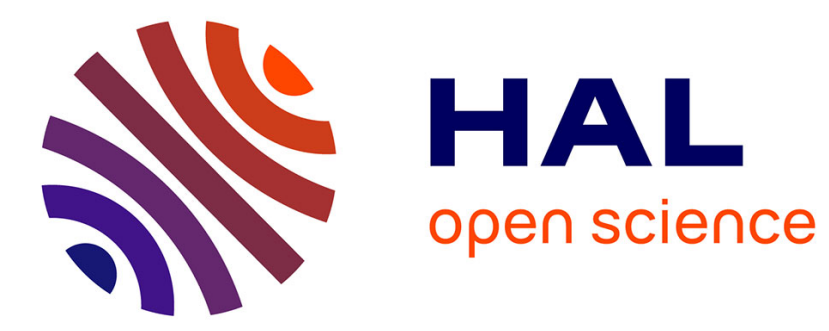

\title{
Associations entre récepteurs opiö̈des : vers de nouvelles stratégies thérapeutiques pour la douleur et l'addiction
}

\author{
Marion Gaborit, Dominique Massotte
}

\section{To cite this version:}

Marion Gaborit, Dominique Massotte. Associations entre récepteurs opiö̈des: vers de nouvelles stratégies thérapeutiques pour la douleur et l'addiction. Douleur et Analgésie, 2019, 32 (2), pp.111112. 10.3166/dea-2019-0057 . hal-02511618

\section{HAL Id: hal-02511618 \\ https://hal.science/hal-02511618}

Submitted on 18 Mar 2020

HAL is a multi-disciplinary open access archive for the deposit and dissemination of scientific research documents, whether they are published or not. The documents may come from teaching and research institutions in France or abroad, or from public or private research centers.
L'archive ouverte pluridisciplinaire HAL, est destinée au dépôt et à la diffusion de documents scientifiques de niveau recherche, publiés ou non, émanant des établissements d'enseignement et de recherche français ou étrangers, des laboratoires publics ou privés. 
Associations entre récepteurs opiö̈des : vers de nouvelles stratégies thérapeutiques pour la douleur et l'addiction

Association between opioids receptors: toward new therapeutic strategies for pain and addiction

Mots clés : récepteurs opioïdes, hétéromères, RCPG, douleur, nociception, addiction, mu, delta, kappa

Keywords: opioid receptors; heteromer; GPCR; pain; nociception; addiction; mu; delta; kappa.

Résumé : Les récepteurs opioïdes modulent de nombreuses fonctions physiologiques. Ces récepteurs peuvent s'associer entre eux pour former une nouvelle entité fonctionnelle nommée hétéromère doté de propriétés fonctionnelles spécifiques. Des études in-vivo ont révélé que ces hétéromères jouaient un rôle crucial dans la douleur aigué et chronique ainsi que dans l'addiction, les désignant alors comme une nouvelle cible thérapeutique pour le traitement de ces pathologies.

Abstract: Opioid receptors modulate numerous physiological functions. These receptors can associate each other to form a new functional entity named heteromer which possess specific functional properties. In-vivo data suggest a crucial role for opioid heteromers in acute or chronic pain as well as addiction, pointing them as a new therapeutic target for the treatment of these pathologies.

Marion Gaborit*1, Dominique Massotte ${ }^{1}$

${ }^{1}$ Centre National de la recherche Scientifique et Université de Strasbourg, Institut des Neurosciences Cellulaires et Intégratives (CNRS UPR 3212), Strasbourg France

\section{Adresse de correspondance (*)}

Mme Gaborit Marion, Equipe Neuroanatomie, douleur \& psychopathologies, Institut des Neurosciences Cellulaires et Intégratives (INCI - CNRS UPR 3212), 8 Allée du Général Rouvillois 67000 Strasbourg

Tel : 0388456652 / Email : marion.gaborit@etu.unistra.fr; d.massotte@unistra.fr 
Informations sur l'auteur principal, les co-auteurs et sur le laboratoire d'appartenance Mme Marion Gaborit est étudiante en deuxième année du Master de Neurosciences Cellulaires et Intégrées de Strasbourg. Son projet de stage est de déterminer les effets d'un traitement à la morphine sur la signalisation et l'internalisation hétéromères opiö̈des mudelta. Ce stage est réalisé sous la supervision du Dr Dominique Massotte, directrice de recherche CNRS.

\section{Texte (3702 caractères)}

Le système opioïde intervient dans la modulation de nombreuses fonctions biologiques, telles que nociception, récompense aux stimuli naturels, émotions, humeur, stress, fonctions autonomes, mémoire ou encore immunité. Il agit à travers trois récepteurs mu, delta et kappa couplés à des protéines $G$ inhibitrices qui sont la cible de peptides endogènes (enképhalines, dynorphines et beta-endorphine). Le récepteur mu est aussi la cible de ligands exogènes tels que la morphine et est responsable de son effet analgésique mais aussi de la tolérance et de la dépendance qui se développent lors de son administration chronique.

En systèmes hétérologues, les récepteurs opiö̈des peuvent s'associer physiquement entre eux pour former une nouvelle entité, nommée hétéromère, qui présente des propriétés fonctionnelles distinctes avec un profil pharmacologique, une signalisation et/ou un trafic intracellulaire différents de ceux des récepteurs seuls [1]. In vivo, le rôle fonctionnel des hétéromères mu-delta, mu-kappa et delta-kappa reste cependant très largement méconnu.

Les hétéromères mu-kappa ont été uniquement décrit dans la moelle épinière chez la ratte en période de pré-œstrus où leur co-activation par la dynorphine et la morphine entraîne une augmentation de l'analgésie spinale induite par la morphine [2]. La présence d'hétéromères delta-kappa a quant à elle été établie dans les neurones sensoriels primaires de 
rats $[3,4]$ où leur ciblage sélectif produit une analgésie thermique durable résultant de leur interaction fonctionnelle $[4,5]$.

Les hétéromères mu-delta ont pour leur part été davantage étudiés. La co-expression neuronale des récepteurs mu et delta est présente dans les circuits associés à la perception et au traitement des stimuli aversifs dont la nociception [6]. De plus, la proximité physique entre les deux récepteurs a été établie dans le système nerveux central et périphérique [6-10]. De façon intéressante, un traitement chronique à la morphine entraine une augmentation du nombre de récepteurs delta à la surface membranaire [11-13] et accroit la co-expression neuronale des récepteurs mu et delta dans le cerveau où elle apparait dans des régions associées à la récompense mais aussi essentielles au contrôle viscéral et au traitement émotionnel du sevrage [14]. De plus, ceci s'accompagne d'une augmentation de la densité d'hétéromères mu-delta dans des régions clés de ces circuits [10]. La persistance de ces changements à l'état abstinent suggère que les hétéromères mu-delta jouent un rôle capital dans la rechute et l'hyperalgésie induite par la prise chronique d'opiacés [14]. Au niveau moléculaire et cellulaire, l'hétéromérisation entre les deux récepteurs modifie les propriétés pharmacologiques du récepteur mu, sa signalisation et son devenir intracellulaire [15] mais les conséquences fonctionnelles de ces changements restent mal comprises. En effet, ils semblent contribuer au développement de la tolérance morphinique tandis que leur ciblage spécifique induit une forte analgésie associée à une faible tolérance et dépendance [15].

En conclusion, les résultats obtenus à ce jour démontrent une implication des hétéromères entre récepteurs opioïdes dans la modulation de la nociception mais aussi un rôle complexe au niveau de l'analgésie induite par la morphine. Cependant, ils les désignent avant tout comme des cibles thérapeutiques à part entière pouvant induire une réponse analgésique avec un développement moindre de la tolérance et/ou dépendance comparé à la morphine. 
Des études plus approfondies sont donc maintenant requises pour établir de façon précise le rôle fonctionnel de ces hétéromères et leur contribution aux états physiopathologiques.

Liens d'intérêts: les auteurs déclarent ne pas avoir de lien d'intérêt

\section{Références:}

1. Pin J P, Neubig R, Bouvier M et al (2007) International Union of Basic and Clinical Pharmacology. LXVII. Recommendations for the recognition and nomenclature of G proteincoupled receptor heteromultimers. Pharmacol Rev 59:5-13

2. Chakrabarti S, Liu N J and Gintzler A R (2010) Formation of mu-/kappa-opioid receptor heterodimer is sex-dependent and mediates female-specific opioid analgesia. Proc Natl Acad Sci U S A 107:20115-9

3. Berg K A, Rowan M P, Gupta A et al (2012) Allosteric interactions between delta and kappa opioid receptors in peripheral sensory neurons. Mol Pharmacol 81:264-72

4. Jacobs B A, Pando M M, Jennings E et al (2018) Allosterism within delta Opioid-kappa Opioid Receptor Heteromers in Peripheral Sensory Neurons: Regulation of kappa Opioid Agonist Efficacy. Mol Pharmacol 93:376-86

5. Jacobs B A, Pando M M, Jennings E M et al (2019) Signaling characteristics and functional regulation of delta opioid-kappa opioid receptor (DOP-KOP) heteromers in peripheral sensory neurons. Neuropharmacology

6. Erbs E, Faget L, Scherrer G et al (2015) A mu-delta opioid receptor brain atlas reveals neuronal co-occurrence in subcortical networks. Brain Struct Funct 220:677-702

7. Kabli N, Nguyen T, Balboni G et al (2013) Antidepressant-like and anxiolytic-like effects following activation of the mu-delta opioid receptor heteromer in the nucleus accumbens. Mol Psychiatry 19:968-94

8. Xie W Y, He Y, Yang Y R et al (2009) Disruption of Cdk5-associated phosphorylation of residue threonine-161 of the delta-opioid receptor: impaired receptor function and attenuated morphine antinociceptive tolerance. J Neurosci 29:3551-64

9. He S Q, Zhang Z N, Guan J S et al (2011) Facilitation of mu-opioid receptor activity by preventing delta-opioid receptor-mediated codegradation. Neuron 69:120-31

10. Gupta A, Mulder J, Gomes I et al (2010) Increased abundance of opioid receptor heteromers after chronic morphine administration. Sci Signal 3:ra54

11. Gendron L, Mittal N, Beaudry H et al (2015) Recent advances on the delta opioid receptor: from trafficking to function. Br J Pharmacol 172:403-19

12. Ong E W, Xue L, Olmstead M C et al (2015) Prolonged morphine treatment alters delta opioid receptor post-internalization trafficking. Br J Pharmacol 172:615-29

13. Erbs E, Faget L, Ceredig R A et al (2016) Impact of chronic morphine on delta opioid receptor-expressing neurons in the mouse hippocampus. Neuroscience 313:46-56

14. Pierre F, Ugur M, Faivre F et al (2019) Morphine-dependent and abstinent mice are characterized by a broader distribution of the neurons co-expressing mu and delta opioid receptors. Neuropharmacology pii: S0028-3908(19)30086-3

15. Ugur M, Derouiche L and Massotte D (2018) Heteromerization Modulates mu Opioid Receptor Functional Properties in vivo. Front Pharmacol 9:1240 